\title{
AN ISOGEOMETRIC SOLID-SHELL MODEL FOR POSTBUCKLING OPTIMISATION STRATEGIES
}

\section{FRANCESCO S. LIGUORI ${ }^{1}$, ANTONIO MADEO ${ }^{1}$, LEONARDO LEONETTI ${ }^{1}$, DOMENICO MAGISANO ${ }^{1}$ AND GIOVANNI GARCEA ${ }^{1}$}

\author{
${ }^{1}$ Dipartimento di Ingegneria Informatica, Modellistica, Elettronica e Sistemistica \\ Università della Calabria, Italy, francesco.liguori@unical.it
}

Key words: Isogeometry, postbuckling, imperfection sensitivity, optimisation

\begin{abstract}
The optimal design of shell structures undergoing buckling phenomena is nowadays an open problem, whose solution would provide interesting answers to both academy and industry. The main difficulty is represented by the high computational cost required for optimising a full-scale structure characterised by a relatively complex postbuckling behaviour. In this work, this topic is addressed by proposing an isogeometric solid-shell model. The equilibrium path is evaluated through a multimodal Koiter's method. The resulting postbuckling analysis turns out to be efficient and accurate, as shown by numerical examples.
\end{abstract}

\section{INTRODUCTION}

Composite shells are nowadays employed as primary structural elements in a very wide range of applications $[1,2]$. Their success is mainly due to the high strength/weight ratio that is crucial for lightweight structures. The failure of such elements often occurs because of buckling phenomena which make them sensitive to material, geometrical and load imperfections [3]. Thousands of equilibrium path evaluations can be required in order to detect the worst imperfection case in terms of failure load. The arc-length nonlinear analysis is commonly used to reconstruct the equilibrium path of such structures. Although this method easily provides the desired information for assigned data, it is too time consuming and inappropriate for an imperfection sensitivity analysis. Furthermore, if such an analysis is used within optimisation strategies [4], its computational cost could become unfeasible. For these reasons, a great amount of research has focused on developing reduced order models (ROMs) based on the finite element (FE) implementation [5, 6, 7] of the Koiter theory of elastic stability [8]. This numerical strategy, known as Koiter's method (see [9] and references therein), is capable of furnishing, with an acceptable computational cost, an accurate prediction [3] of the limit load value and the initial post-critical behaviour for a very large number of imperfections.

The ROM consists in approximating the unknown fields using the initial path tangent and restricted number $m$ of buckling modes associated to the first buckling loads and the corresponding quadratic correctives. In this way, the response of the structure is defined by a reduced system of nonlinear equations where the unknowns are the load factor and $m$ modal amplitudes and the coefficients correspond to 2 nd, $3 \mathrm{rd}$ and 4th order energy variations. The most convenient aspect of the method is that the effects of geometrical imperfections can be included a posteriori in the reduced system of the perfect structure $[1,2]$. 
A solid-shell FE model [10] proves to be particularly convenient for the construction of the ROM, since it allows us to avoid the use of finite rotations, typical of other exact or corotational shell models, which make the evaluation of the high order strain energy variations complex and expensive. Although a large number of locking free linear solid-shell elements are available, their behaviour is not sufficiently accurate when modelling curved geometries and, as a consequence, a fine discretisation is required. On the other hand, increasing the interpolation order drastically reduces the computational convenience of the element because of the high number of DOFs and the time consuming integration and assembly of the quantities [10].

An interesting alternative is given by the isogeometric analysis (IGA) [11] based on NURBS shape functions. In contrast to Lagrangian functions, one of their feature is the possibility of elevating the order of the interpolation with little change in the number of DOFs. The number of integration points required for the numerical integration is also kept quite low because of the high continuity of the shape functions $[12,13]$. These aspects make IGA very attractive in particular in buckling problems, where a highly continuous solution is often expected and the accurate representation of the geometry is an important requirement for an accurate analysis [3].

Despite the many advantages, some difficulties have to be overcome. Although the use of very high order shape functions eliminates interpolation lockings, the order elevation increases the computational effort. Therefore, $C_{1}$-quadratic and $C_{2}$-cubic NURBS interpolations are often preferred, even though they exhibit locking phenomena. The inter-element high continuity of the interpolation makes it no longer possible to employ element-wise reduced integrations and strategies like Assumed Natural Strain (ANS)

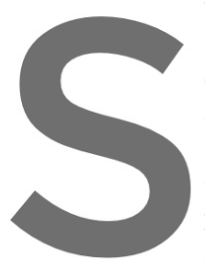
[14] effectively. Conver continuous stress shape with respect to the initial is no longer possible. reduced integration rule in linear elastic problems
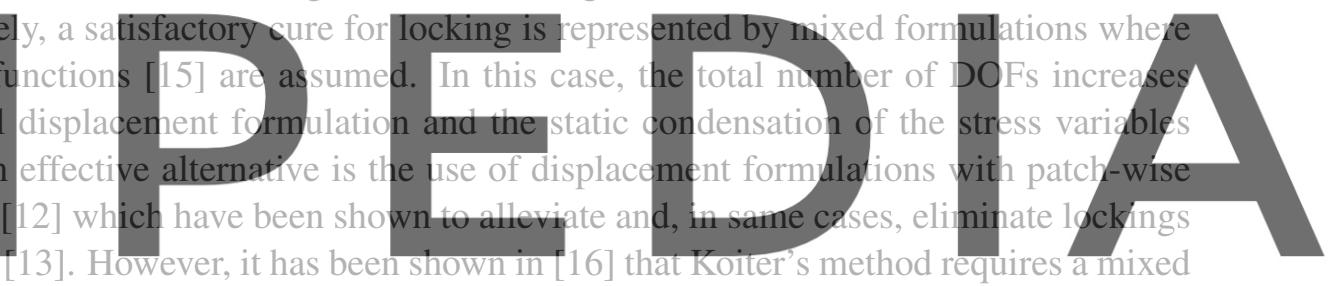

formulation in order to avoid a locking phenomenon in the evaluation of the fourth-order coefficients

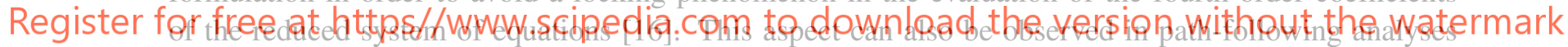

$[16,10]$ where displacement formulations lead to a slow convergence rate of the Newton scheme when slender structures are analysed. This is due to the bad estimate of the stresses when evaluated using extrapolated displacements. In the Koiter analysis this phenomenon is much more evident because the equilibrium path is directly extrapolated using the ROM, and an equilibrium error is not corrected by an iterative scheme, so affecting the accuracy of the method. On the contrary mixed formulations [17] avoid this drawback because the stresses are directly extrapolated.

In this work, we propose an isogeometric numerical formulation of Koiter's theory for the analysis of composite shells which exploits the advantages of a solid-shell model. A linear through-the-thickness interpolation is considered for geometry and displacements. The nonlinear model is based on a TotalLagrangian formulation with the use of the Green-Lagrange strain measure. Inspired by the FE approach proposed by Sze [10], the Green-Lagrange strains are linearised along the thickness direction. The model is described by middle surface coordinates only, allowing us to interpolate geometry and displacements using bivariate NURBS of generic order and continuity. Each control point has six DOFs and only displacement DOFs are employed. Shear and membrane locking, which already occur in linear elastic problems for low order NURBS, are even more evident in the nonlinear range. Different patch-wise 
reduced integration rules [12,13], previously proposed for linear analysis, are investigated in stability problems with the aim of eliminating interpolation lockings and increasing the computational efficiency when $C_{1}$-quadratic and $C_{2}$-cubic NURBS are adopted. To obtain the mixed description of the problem, required by the Koiter formulation, the Mixed Integration Point (MIP) strategy, recently proposed in $[18,19]$ for finite element path-following analyses, is here extended to the proposed isogeometric Koiter analysis. It consists in relaxing the constitutive equations at each integration point, making it possible to rewrite the strain energy of the model in a pseudo Hellinger-Reissner form (see also [20]).

\section{ISOGEOMETRIC SOLID-SHELL MODEL}

An isogeometric solid-shell model for Koiter analysis of composite shells is derived in this section. Geometry and displacement fields expressed in terms of quantities on the middle surface of the shell are interpolated with NURBS function of generic order [11]. The model, based on Total Lagrangian formulation and Green-Lagrange strain measure, is then derived. It extends the FE model proposed in [10].

\subsection{Shell kinematics}

Convective curvilinear shell coordinates $\xi=[\xi, \eta, \zeta]$ with $(\xi, \eta)$ representing middle surface coordinates and $\zeta$ being the shell thickness coordinate are employed in this Total Lagrangian formulation. The position of material points in the current configuration is given in terms of their position vector $\mathbf{X}(\xi, \eta, \zeta)$ in the reference configuration and the displacement state $\mathbb{d}(\xi, \eta, \zeta)$.
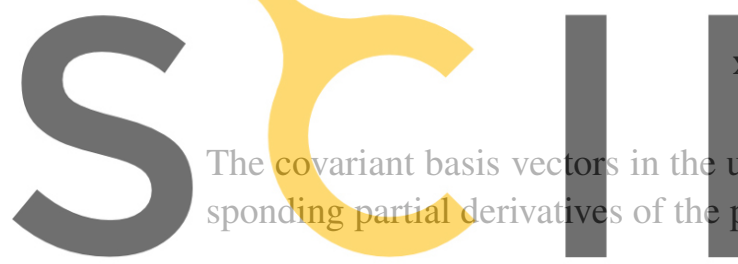

$$
\mathrm{G}_{i}=\mathbf{X}_{, i}
$$
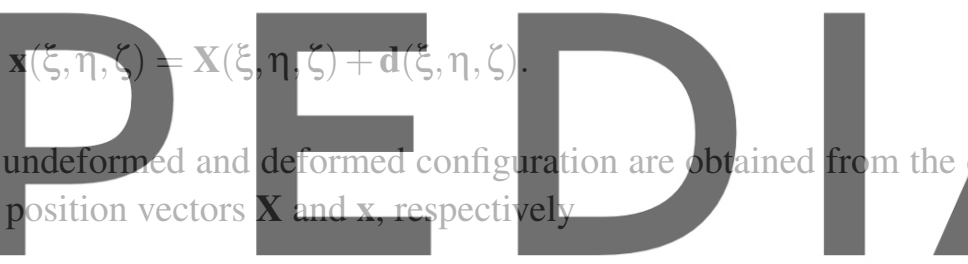

$$
\mathrm{g}_{i}=\mathrm{x}_{, i}=\mathrm{G}_{i}+\mathrm{d}_{, i}
$$

with $i=1,2,3$,

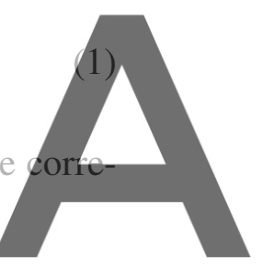

(2)

Register for free at https//www.scipedia.com to download the version without the watermark where ()$_{i}$ denotes the partial derivative with respect to $i$ th components of $\xi$. The contravariant basis vectors follow from the dual basis condition: $\mathrm{g}_{i} \cdot \mathrm{g}^{j}=\mathrm{G}_{i} \cdot \mathrm{G}^{j}=\delta_{i}^{j}$ and the metric coefficients are $g_{i j}=$ $g_{i} \cdot g_{j}$ and $G_{i j}=\mathbf{G}_{i} \cdot \mathbf{G}_{j}$ with $(i, j=1,2,3)$. The motion of material points from the initial reference configuration to the current configuration is described by the deformation map $\mathbf{F}: \mathbf{x} \rightarrow \mathbf{X}$

$$
\mathbf{F}=\frac{\partial \mathbf{x}}{\partial \mathbf{X}}=\mathbf{g}_{i} \otimes \mathbf{G}^{i}
$$

and the Einstein convention of summing on repeated indexes is adopted from now on.

Using the deformation gradient in Eq.(3) and the metric tensor coefficients $g_{i j}$ and $G_{i j}$, the GreenLagrange strain tensor can be expressed as

$$
\mathbf{E}=\frac{1}{2}\left(\mathbf{F}^{T} \mathbf{F}-\mathbf{I}\right)=\bar{E}_{i j} \mathbf{G}^{i} \otimes \mathbf{G}^{j}
$$

with

$$
\bar{E}_{i j}=\frac{1}{2}\left(\mathbf{X}_{i} \cdot \mathbf{d},_{j}+\mathbf{d},_{i} \cdot \mathbf{X},_{j}+\mathbf{d},_{i} \cdot \mathbf{d},_{j}\right) \quad \text { with } \quad i, j=1,2,3
$$


where $(\cdot)$ means scalar product. Assuming a linear through-the-thickness interpolation the position vector is expressed as

$$
\mathbf{X}=\mathbf{X}_{0}[\xi, \eta]+\zeta \mathbf{X}_{n}[\xi, \eta]
$$

where

$$
\mathbf{X}_{0}=\frac{1}{2}(\mathbf{X}[\xi, \eta, 1]+\mathbf{X}[\xi, \eta,-1]), \quad \mathbf{X}_{n}=\frac{1}{2}(\mathbf{X}[\xi, \eta, 1]-\mathbf{X}[\xi, \eta,-1])
$$

with $\zeta=1$ and $\zeta=-1$ identifying the top and the bottom surface of the shell respectively.

Similarly, the displacement field $\mathbf{d}=\mathbf{d}_{0}[\xi, \eta]+\zeta \mathbf{d}_{n}[\xi, \eta]$ is described as a combination of the displacements

$$
\mathbf{d}_{0}=\frac{1}{2}(\mathbf{d}[\xi, \eta, 1]+\mathbf{d}[\xi, \eta,-1]), \quad \mathbf{d}_{n}=\frac{1}{2}(\mathbf{d}[\xi, \eta, 1]-\mathbf{d}[\xi, \eta,-1]) .
$$

The same convective coordinates $\xi$ are used for expressing the interpolation of the discrete model.

\subsection{The isogeometric solid-shell element}

The numerical model is obtained interpolating the geometry and the displacement field using NURBS shape functions.

$$
\mathbf{X}[\boldsymbol{\xi}]=\mathbf{N}_{d}[\boldsymbol{\xi}] \mathbf{X}_{e}, \quad \mathbf{d}[\boldsymbol{\xi}]=\mathbf{N}_{d}[\boldsymbol{\xi}] \mathbf{d}_{e}
$$

where $\mathbf{d}_{e}=\left[\mathbf{d}_{0 e}, \mathbf{d}_{n e}\right]$ and $\mathbf{X}_{e}=\left[\mathbf{X}_{0 e}, \mathbf{X}_{n e}\right]$ collect the element control points for displacement and geometry. The matrix $\mathbf{N}_{d}[\xi]$ collects the interpolation functions
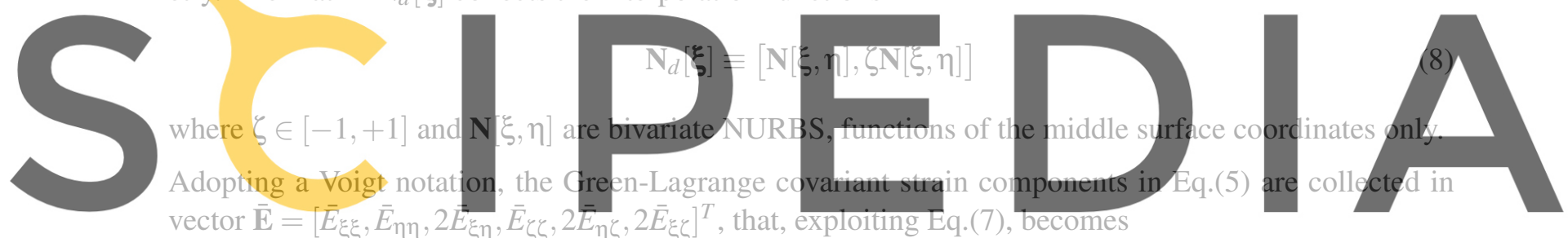

Register for free at https//www.scipedia.com to download the version without the watermark

where $\mathcal{L}[\boldsymbol{\xi}] \equiv Q\left[\xi, \mathbf{X}_{e}\right]$ and $Q$ expression can be found in [21]. By truncation, the linearised covariant strains

$$
\overline{\mathbf{E}} \approx\left[\begin{array}{c}
\overline{\mathbf{e}}[\xi, \eta]+\zeta \bar{\chi}[\xi, \eta] \\
\bar{E}_{\zeta \zeta}[\xi, \eta] \\
\bar{\gamma}[\xi, \eta]
\end{array}\right]
$$

where

$$
\overline{\mathbf{e}}[\xi, \eta] \equiv\left[\begin{array}{c}
\bar{E}_{\xi \xi \xi}[\xi, \eta, 0] \\
\bar{E}_{\eta \eta}[\xi, \eta, 0] \\
2 \bar{E}_{\xi \eta}[\xi, \eta, 0]
\end{array}\right] \quad \bar{\chi}[\xi, \eta] \equiv\left[\begin{array}{c}
\bar{E}_{\xi \xi, \zeta}[\xi, \eta, 0] \\
\bar{E}_{\eta \eta}, \zeta[\xi, \eta, 0] \\
2 \bar{E}_{\xi \eta, \zeta}[\xi, \eta, 0]
\end{array}\right] \quad \bar{\gamma}[\xi, \eta] \equiv\left[\begin{array}{c}
2 \bar{E}_{\eta \zeta}[\xi, \eta, 0] \\
2 \bar{E}_{\xi \zeta}[\xi, \eta, 0]
\end{array}\right]
$$

are collected in the vector of generalized covariant strains $\bar{\varepsilon}[\xi, \eta] \equiv\left[\overline{\mathbf{e}}, \bar{E}_{\zeta \zeta}, \bar{\chi}, \bar{\gamma}\right]^{T}$. In order to simplify the notation, the dependence of the quantities on $\xi, \eta$ will be omitted from now on, when clear. The generalised stress components, once the kinematic model is assumed, are automatically given by 
assuring the invariance of the internal work. By collecting the contravariant stress components $\overline{\mathbf{S}} \equiv$ $\left[\bar{S}_{\xi \xi}, \bar{S}_{\eta \eta}, \bar{S}_{\xi \eta}, \bar{S}_{\zeta \zeta}, \bar{S}_{\eta \zeta}, \bar{S}_{\xi \zeta}\right]^{T}$, the work conjugate variables with $\bar{\varepsilon}$ are obtained by

$$
\mathcal{W}=\int_{V} \overline{\mathbf{S}}^{T} \overline{\mathbf{E}} d V=\int_{\Omega}\left(\overline{\mathcal{N}}^{T} \overline{\mathbf{e}}+\overline{\mathcal{M}}^{T} \bar{\chi}+\bar{s}_{\zeta \zeta} \bar{E}_{\zeta \zeta}+\overline{\mathcal{T}}^{T} \bar{\gamma}\right)=\int_{\Omega} \bar{\sigma}^{T} \bar{\varepsilon} d \Omega
$$

where, from now on, $\int_{\Omega}(\ldots) \Omega=2 \int_{\xi_{i}}^{\xi_{i+1}} \int_{\eta_{i}}^{\eta_{i+1}}(\ldots) \operatorname{det}(\mathbf{J}[\xi, \eta, 0]) d \xi d \eta$ and $\mathbf{J}$ denotes the Jacobian matrix $\mathbf{J}[\xi, \eta, \zeta]=\left[\mathbf{G}_{1}, \mathbf{G}_{2}, \mathbf{G}_{3}\right]^{T}$.

The generalized contravariant stresses $\bar{\sigma} \equiv\left[\overline{\mathcal{N}}, \overline{s_{\zeta \zeta}}, \overline{\mathcal{M}}, \overline{\mathcal{T}}\right]^{T}$ in Eq.(11) are then

$$
\overline{\mathcal{N}} \equiv \frac{1}{2} \int_{-1}^{1} \bar{\sigma}_{p} d \zeta \quad \overline{\mathcal{M}} \equiv \frac{1}{2} \int_{-1}^{1} \zeta \bar{\sigma}_{p} d \zeta \quad \bar{s}_{\zeta \zeta} \equiv \frac{1}{2} \int_{-1}^{1} \bar{S}_{\zeta \zeta} d \zeta \quad \overline{\mathcal{T}} \equiv \frac{1}{2} \int_{-1}^{1} \bar{\tau} d \zeta
$$

with

$$
\bar{\sigma}_{p}=\left[\begin{array}{l}
\bar{S}_{\xi \xi} \\
\bar{S}_{\eta \eta} \\
\bar{S}_{\xi \eta}
\end{array}\right] \quad \bar{\tau}=\left[\begin{array}{l}
\bar{S}_{\xi \zeta} \\
\bar{S}_{\eta \zeta}
\end{array}\right] .
$$

\subsection{The mapping to the physical domain}

The relation between the contravariant stresses and covariant strains in tensor notation and the corresponding Cartesian ones is
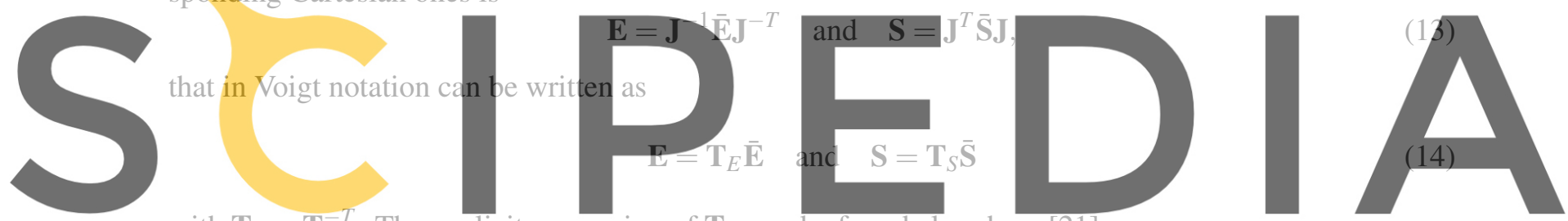

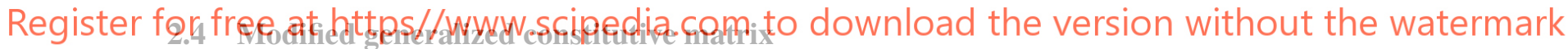

The homogenization technique proposed in [10] is employed. A modified generalized constitutive matrix

$\mathbf{C}_{\varepsilon}$ is derived by integrating the constitutive law of each lamina and assuming a constant with $\zeta$ stress $S_{\zeta \zeta}$ through the layers in order to eliminate thickness locking. In this way an accurate prediction of the global behavior in terms of stresses and displacements is obtained.

The thickness locking free generalized constitutive law in the global reference system is

$$
\sigma[\xi, \eta]=\mathbf{C}_{\varepsilon} \varepsilon[\xi, \eta]
$$

Alternatively, the constitutive matrix can be expressed as a function of lamination parameters that is useful in optimisation problems, as shown in [2].

\section{KOITER IGA}

The standard Koiter approach, described in detail in[3], is now briefly recalled and the steps of the algorithms are particularised to IGA using the MIP strategy and the patch-wise integration. 


\subsection{The nonlinear model and the numerical integration}

We consider a slender hyperelastic structure subject to conservative loads $p[\lambda]$ proportionally increasing with the amplifier factor $\lambda$. The equilibrium is expressed by the virtual work equation

$$
\Phi[u]^{\prime} \delta u-\lambda \hat{p} \delta u=0 \quad, \quad u \in \mathcal{U}, \delta u \in \mathcal{T}
$$

where $u \in \mathcal{U}$ is the field of configuration variables, $\Phi[u]$ denotes the strain energy, $\mathcal{T}$ is the tangent space of $\mathcal{U}$ at $u$ and a prime is used to express the Frechèt derivative with respect to $u$. We assume that $\mathcal{U}$ will be a linear manifold so that its tangent space $\mathcal{T}$ will be independent of $u$. When a mixed format is adopted the configuration variables $u$ collect both displacement and stress fields.

The displacement based IGA formulation previously presented allows us to express the strain energy of the element as a sum of element contributions $\Phi[u] \equiv \sum_{e} \Phi_{e}\left[\mathbf{d}_{e}\right]$

$$
\Phi_{e}\left[\mathbf{d}_{e}\right] \equiv \int_{\Omega_{e}}\left(\frac{1}{2} \varepsilon^{T} \mathbf{C}_{\varepsilon} \varepsilon\right) d \Omega_{e}
$$

where $\Omega_{e}$ is the element domain and a numerical integration is usually adopted.

\subsubsection{Patch-wise reduced integrations}

Recently, patch-wise integration rules, which take into account the inter-element high continuity of the displacement interpolation have been proposed $[13,12]$ and applied to linear elastic problems. The $d$ -

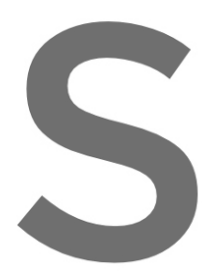
dimensional target space, matrix), of order $p$ and $r$ integration points per elen the splines and $r$ is the gon The patch-wise integration of a given space $S_{r}^{P}$ als
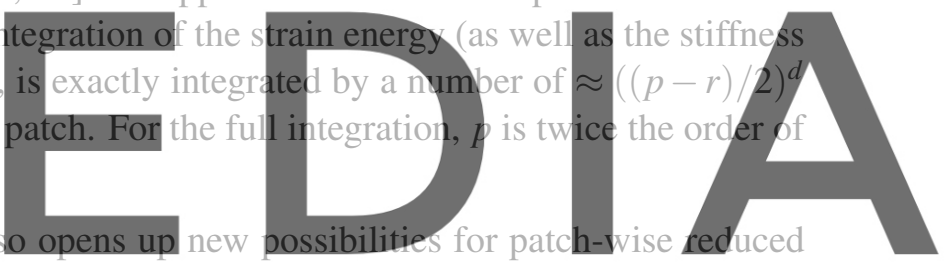

integration schemes. In fact $p$ and $r$ can be selected by the user and are not required to be those for the

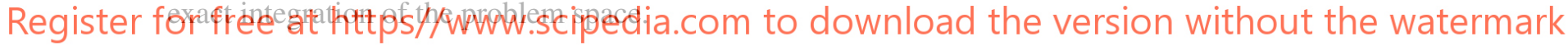

Being that in patch-wise rules the number of integration points $n$ can be different element-by-element, the strain energy can then be evaluated as

$$
\Phi_{e}\left[\mathbf{d}_{e}\right] \equiv \frac{1}{2} \sum_{g=1}^{n} \varepsilon_{g}\left[\mathbf{d}_{e}\right]^{T} \mathbf{C}_{g} \varepsilon_{g}\left[\mathbf{d}_{e}\right] w_{g}
$$

where subscript $g$ denotes quantities evaluated at the integration point $\left[\xi_{g}, \eta_{g}\right], w_{g}$ is the product of the corresponding weight and the determinant of the Jacobian matrix $\mathbf{J}$ evaluated at the integration point and $\mathbf{C}_{g}$ is $\mathbf{C}_{\varepsilon}$ at the integration point.

\subsection{The mixed integration point strategy}

The fundamental idea of MIP strategy is to relax the constitutive equations at the level of each integration point. This is made by rewriting the strain energy in a pseudo Hellinger-Reissner form on the element

$$
\Phi_{e}\left[\mathbf{u}_{e}\right] \equiv \sum_{g=1}^{n}\left(\sigma_{g}^{T} \varepsilon_{g}\left[\mathbf{d}_{e}\right]-\frac{1}{2} \sigma_{g}^{T} \mathbf{C}_{g}^{-1} \sigma_{g}\right) w_{g}
$$


where the stresses at each integration point $\sigma_{g}$ are now independent variables being

$$
\mathbf{u}_{e}=\left[\sigma_{1}, \ldots, \sigma_{n}, \mathbf{d}_{e}\right]^{T}
$$

From the stationary condition with respect to $\sigma_{g}$ we obtain the constitutive law at the integration point $g$

$$
\mathbf{s}_{g \sigma} \equiv \varepsilon_{g}\left[\mathbf{d}_{e}\right]-\mathbf{C}_{g}^{-1} \sigma_{g}
$$

that, if substituted in Eq.(19), again furnishes the displacement formulation in Eq.(18).

\subsection{The implementation of the Koiter method using mixed integration points}

The asymptotic approach is based on a third order Taylor expansion of Eq.(16), in terms of load factor $\lambda$ and modal amplitudes $\alpha_{i}$. We refer readers to [3] for recent developments of the method and more detail.

Letting $u_{i} \in \mathcal{T}$ be a generic variation of the displacement field and denoting with a bold symbol the discrete FEM counterpart of the continuum quantities, and referring to the solid-shell finite element model presented in [9], the construction of the reduced model of the perfect structure consists of the following steps.

\section{The fundamental path is evaluated as}

$$
\mathbf{u}^{f}[\lambda]=\mathbf{u}_{0}+\lambda \hat{\mathbf{u}}, \quad \mathbf{K}_{0} \hat{\mathbf{u}}=\mathbf{f}, \quad \mathbf{K}_{0} \equiv \mathbf{K}\left[\mathbf{u}_{0}\right]
$$
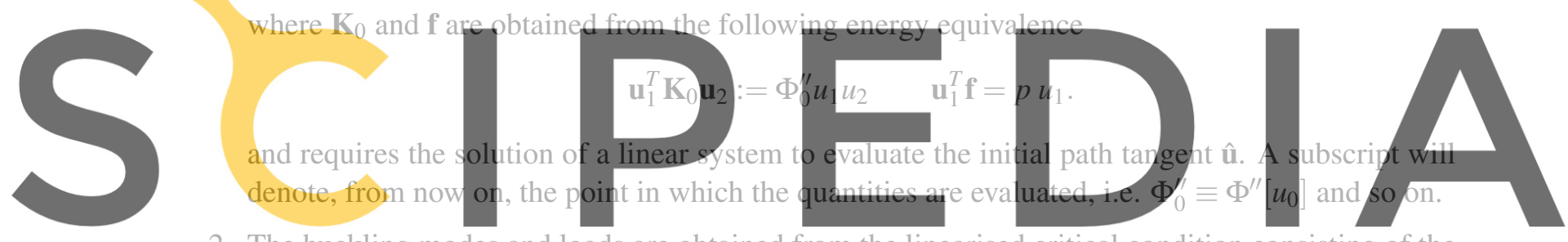

2. The buckling modes and loads are obtained from the linearised critical condition consisting of the

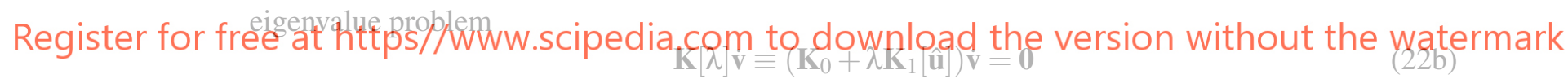

where $\mathbb{K}_{1}$ is obtained from the following energy equivalence

$$
\mathbf{u}_{1}^{T} \mathbf{K}_{1} \mathbf{u}_{2}=\Phi_{0}^{\prime \prime \prime} \hat{u} u_{1} u_{2}
$$

3. The $(m \times(m+1)) / 2+1$ quadratic corrective FE vectors $\mathbf{w}_{i j}, \hat{\hat{\mathbf{w}}}$ are obtained by the solution of the linear systems

$$
\begin{array}{lll}
\mathbf{K}_{b} \hat{\hat{\mathbf{w}}}+\hat{\mathbf{f}}+\sum_{k=1}^{m} c_{k} \hat{\mathbf{f}}_{k}=\mathbf{0} & \text { with } \quad c_{k}=\dot{\mathbf{v}}_{k}^{T} \hat{\hat{\mathbf{f}}} \\
\mathbf{K}_{b} \mathbf{w}_{i j}+\mathbf{f}_{i j}+\sum_{k=1}^{m} c_{k} \hat{\mathbf{f}}_{k}=\mathbf{0} & \text { with } \quad c_{k}=\dot{\mathbf{v}}_{k}^{T} \mathbf{f}_{i j}
\end{array}
$$

in which $\mathbf{K}_{b} \equiv \mathbf{K}_{0}+\lambda_{b} \mathbf{K}_{1}, \hat{\mathbf{f}}_{k}=\mathbf{K}_{1} \dot{\mathbf{v}}_{k}, \lambda_{b}$ is a reference value of the bifurcation cluster, usually the first buckling load and $\mathbf{f}_{i j}, \mathbf{f}_{00}$ are defined as a function of modes $\dot{\mathbf{v}}_{i}$ and $\hat{\mathbf{u}}$ by the energy equivalences

$$
\delta \mathbf{w}^{T} \mathbf{f}_{i j}=\Phi^{\prime \prime \prime} \dot{v}_{j} \dot{v}_{j} \delta w \quad \delta \mathbf{w}^{T} \hat{\mathbf{f}}=\Phi^{\prime \prime \prime} \hat{u}^{2} \delta w
$$




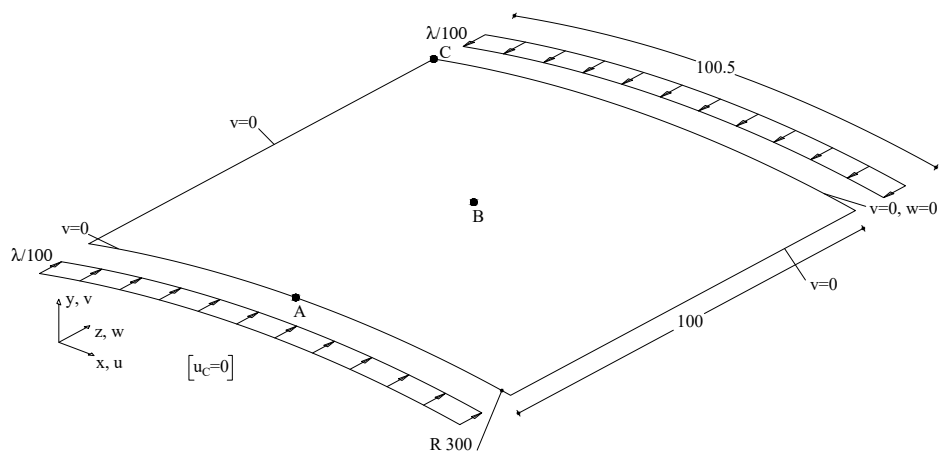

Figure 1: Composite curved panel: geometry and boundary conditions.

4. The construction of the reduced system of equations $(k=1 \ldots m)$

$$
r_{k}\left[\lambda, \alpha_{i}\right] \equiv \mu_{k}[\lambda]+\left(\lambda_{k}-\lambda\right) \alpha_{k}-\frac{1}{2} \lambda^{2} \sum_{i=1}^{m} \alpha_{i} C_{i k}+\frac{1}{2} \sum_{i, j=1}^{m} \alpha_{i} \alpha_{j} \mathcal{A}_{i j k}+\frac{1}{6} \sum_{i, j, h=1}^{m} \alpha_{i} \alpha_{j} \alpha_{h} \mathcal{B}_{i j h k}=0 \quad(22 \mathrm{~d})
$$

is carried out by evaluating the energy terms for $i, j, h, k=1 \cdots m$ as sum of element contributions

$$
\mathcal{A}_{i j k}=\Phi^{\prime \prime \prime} \dot{v}_{i} \dot{v}_{j} \dot{v}_{k}, \quad C_{i k}=\Phi_{b}^{\prime \prime} \hat{\hat{w}} w_{i k}
$$
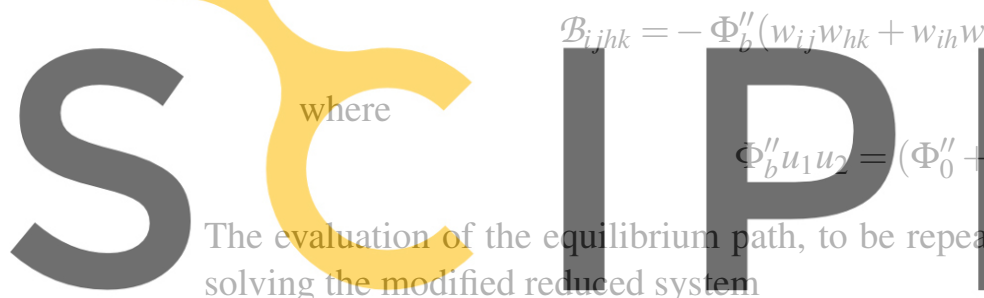

solving the modified reduced system
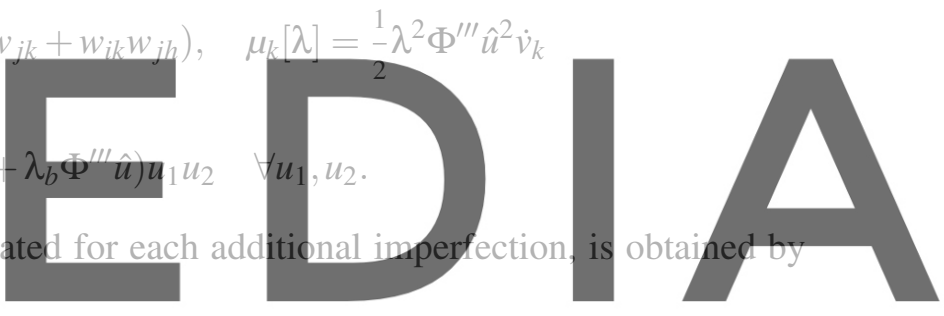

Register for free at https//www.scipedia.com to down $r_{k}\left[\lambda, \alpha_{i}\right]+\tilde{\mu}_{k}\left[\lambda, \alpha_{i}\right]=0$ ad the version without the watermark where $\tilde{\mu}_{k}$ represents the effect of the imperfection, and can be evaluated as in [3].

\section{NUMERICAL RESULTS}

The proposed numerical tool is now tested in a common composite structure. The test regards a curved panel under compression whose geometry, loads, and boundary conditions are represented in Fig.1. The postbuckling response of this panel has been optimised in [4, 2]. The material properties are $E_{11}=30.6$, $E_{22}=E_{33}=8.7, v_{12}=v_{13}=0.29, v_{23}=0.5, G_{12}=G_{13}=3.24, G_{23}=2.9$, while the stacking sequence is $[45,-45,0]_{s}$. Tables 1 and 2 show the convergence of the first 4 linearised buckling loads. The $\bar{S}_{0}^{2}$ for $C_{1}$ and $S_{1}^{4}$ and $\bar{S}_{1}^{3}$ for $C_{2}$ represent the best choices in terms of accuracy and efficiency.

The study of the initial post-buckling behavior of the panel is carried out considering the presence of a geometrical imperfection $\tilde{e}$ that is a combination of the first and the second buckling modes. In particular, it is the difference between them scaled in order to obtain $\|\tilde{e}\|_{\infty}=0.1 t$. Four buckling modes are used for constructing the ROM. The initial post-buckling exhibits a limit load, as shown in in Fig.2 and $3 . C_{1}-\bar{S}_{0}^{2}$, $C_{2}-\bar{S}_{1}^{3}$ and $C_{2}-S_{1}^{4}$ are the best performing strategies, providing a good estimate of the limit loads with a $8 \times 8$ mesh, which became practically exact using a $16 \times 16$ mesh. 


\begin{tabular}{|c|c|c|c|c|c|c|c|c|c|c|c|}
\hline \multicolumn{4}{|l|}{4 elm. } & \multicolumn{4}{|l|}{8 elm. } & \multicolumn{4}{|c|}{16 elm. } \\
\hline $\bar{S}_{0}^{2}$ & $S_{0}^{3}$ & $S_{0}^{4}$ & ANS & $\bar{S}_{0}^{2}$ & $S_{0}^{3}$ & $S_{0}^{4}$ & ANS & $\bar{S}_{0}^{2}$ & $S_{0}^{3}$ & $S_{0}^{4}$ & ANS \\
\hline 1.096 & 1.144 & 1.263 & 1.165 & 1.016 & 1.018 & 1.095 & 1.054 & 1.001 & 1.002 & 1.015 & 1.004 \\
\hline 1.082 & 1.201 & 1.656 & 1.106 & 1.013 & 1.010 & 1.078 & 1.036 & 0.998 & 0.999 & 1.011 & 1.001 \\
\hline 1.080 & 1.346 & $*$ & 1.333 & 1.007 & 1.010 & 1.157 & 1.061 & 0.998 & 0.999 & 1.010 & 0.999 \\
\hline 1.235 & 1.456 & $*$ & 1.848 & 1.035 & 1.073 & 1.228 & 1.119 & 1.003 & 1.012 & 1.049 & 1.011 \\
\hline
\end{tabular}

Table 1: Composite curved panel: first 4 normalized buckling loads for $[45,-45,0]_{s}$ with $C_{1}$ interpolation.

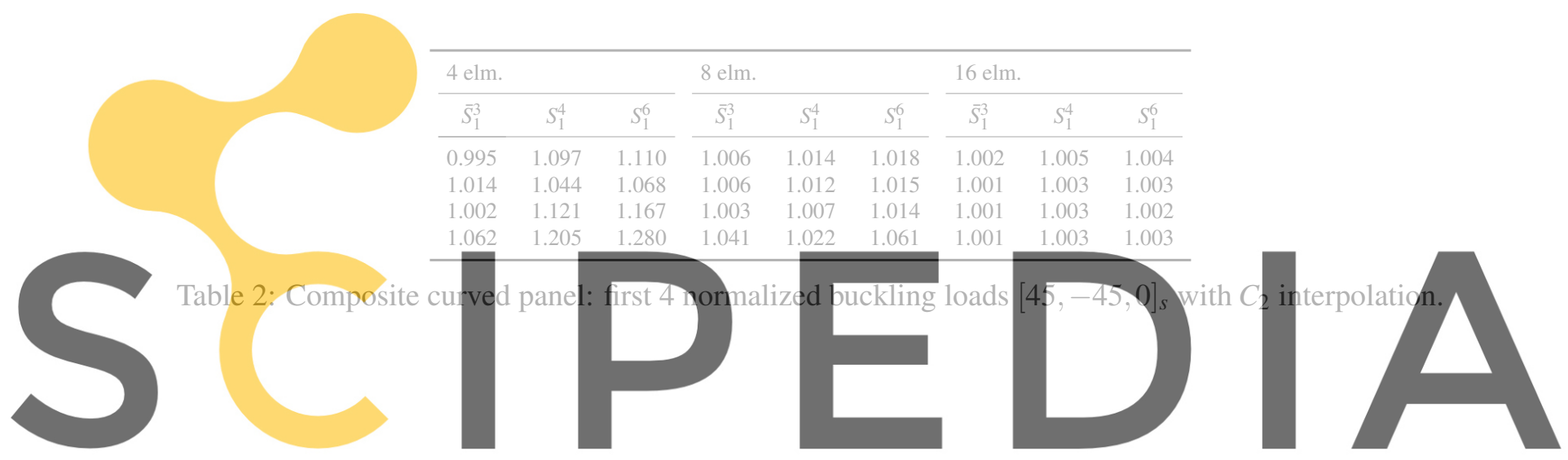

Register for free at https//www.scipedia.com to downłoad the version without the watermark

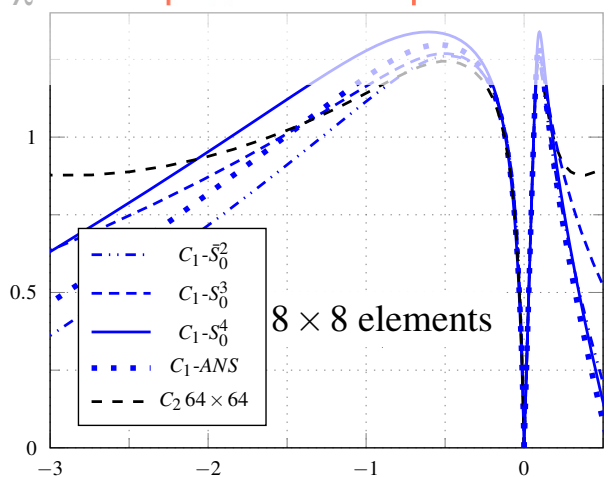

$\mathrm{u}$

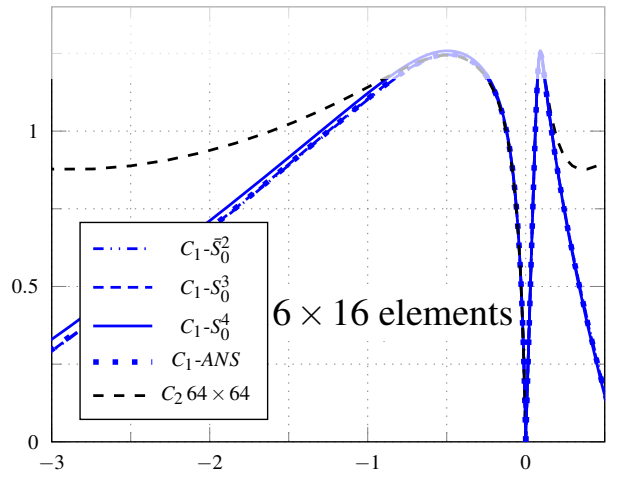

$\mathrm{u}$

Figure 2: Composite curved panel: equilibrium path for $[45 /-45 / 0]$ and $C_{1}$ interpolation 
$\lambda$

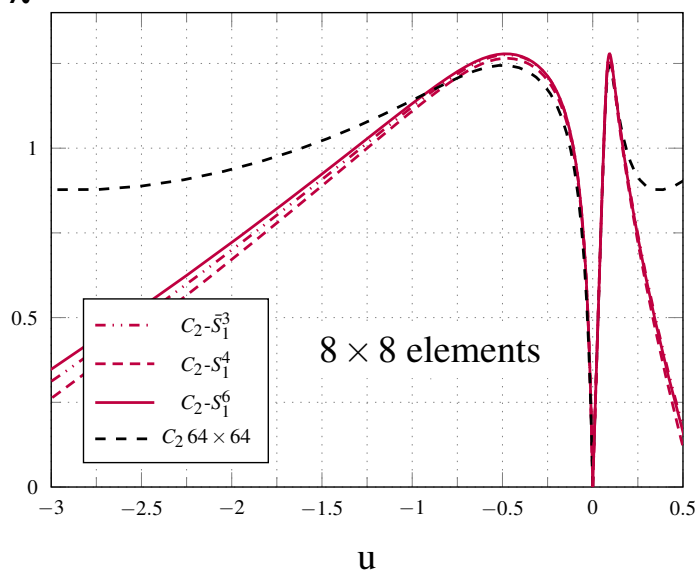

$\lambda$

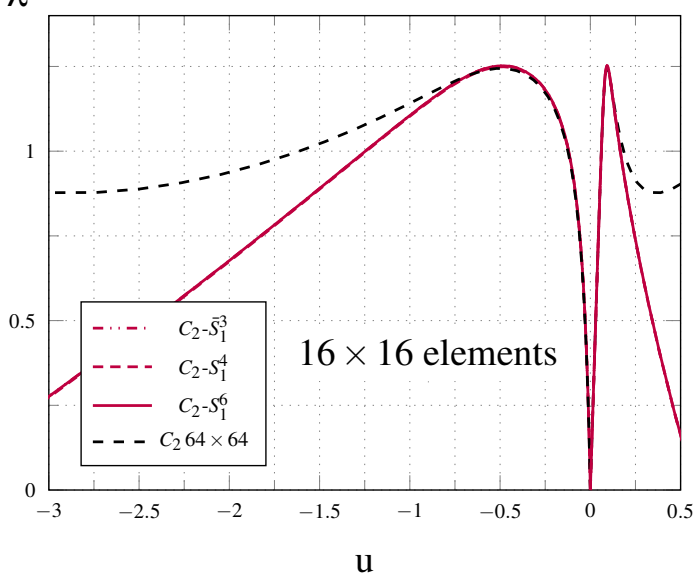

Figure 3: Composite curved panel: equilibrium path for $[45 /-45 / 0]$ and $C_{2}$ interpolation

\section{CONCLUSIONS}

In this work an isogeometric version of Koiter's method has been proposed. The main purpose was to develop a numerical tool to be used within postbuckling optimisation problems. A solid-shell structural model is adopted and Green-Lagrange strain measures are used. A Mixed Integration Point formulation has been used in order t

On the other hand, interpo thereby allowing low orden confirmed that the proposed tool

REFERENCES
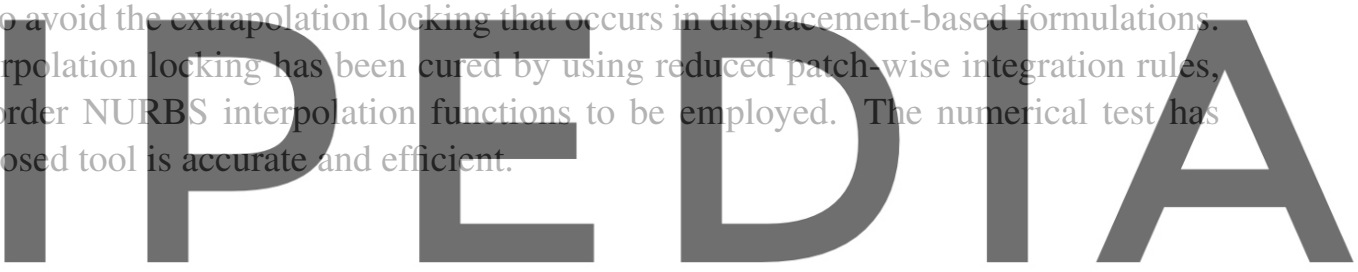

[1] F. S. Liguori, G. Zucco, A. Madeo, D. Magisano, L. Leonetti, G. Garcea, P. M. Weaver, Postbuck-

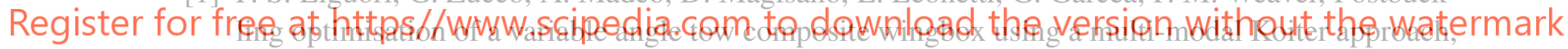
Thin-Walled Structures 138 (2019) 183-198. doi:https://doi.org/10.1016/j.tws.2019.01.035.

[2] F. S. Liguori, G. Zucco, A. Madeo, G. Garcea, L. Leonetti, P. M. Weaver, An isogeometric framework for the optimal design of variable stiffness shells undergoing large deformations, International Journal of Solids and Structures (2020). doi:https://doi.org/10.1016/j.ijsolstr.2020.11.003. URL http://www.sciencedirect.com/science/article/pii/S0020768320304261

[3] G. Garcea, F. Liguori, L. Leonetti, D. Magisano, A. Madeo, Accurate and efficient a posteriori account of geometrical imperfections in Koiter finite element analysis, International Journal for Numerical Methods in Engineering (2017). doi:10.1002/nme.5550.

[4] F. S. Liguori, A. Madeo, D. Magisano, L. Leonetti, G. Garcea, Post-buckling optimisation strategy of imperfection sensitive composite shells using Koiter method and Monte Carlo simulation, Composite Structures (2018). doi:https://doi.org/10.1016/j.compstruct.2018.03.023.

URL http://www.sciencedirect.com/science/article/pii/s0263822317339776

[5] E. Boutyour, H. Zahrouni, M. Potier-Ferry, M. Boudi, Asymptotic-numerical method for buckling 
analysis of shell structures with large rotations, Journal of Computational and Applied Mathematics 168 (1-2) (2004) 77-85. doi:10.1016/j.cam.2003.05.010.

[6] N. Silvestre, D. Camotim, Asymptotic-numerical method to analyze the postbuckling behavior, imperfection-sensitivity, and mode interaction in frames, Journal of Engineering Mechanics 131 (6) (2005) 617-632. doi:10.1061/(ASCE)0733-9399(2005)131:6(617).

[7] B. Schafer, L. Graham-Brady, Stochastic post-buckling of frames using Koiter's method, International Journal of Structural Stability and Dynamics 6 (3) (2006) 333-358. doi:10.1142/S0219455406001976.

[8] W. Koiter, On the stability of elastic equilibrium, english transl. nasa tt-f10, 883 (1967) and affdltr70-25 (1970) Edition, Techische Hooge School at Delft, 1945.

[9] D. Magisano, L. Leonetti, G. Garcea, Koiter asymptotic analysis of multilayered composite structures using mixed solid-shell finite elements, Composite Structures 154 (2016) 296-308. doi:10.1016/j.compstruct.2016.07.046.

[10] K. Sze, W. Chan, T. Pian, An eight-node hybrid-stress solid-shell element for geometric non-linear analysis of elastic shells, International Journal for Numerical Methods in Engineering 55 (7) (2002) 853-878. doi:10.1002/nme.535.

[11] Y. B. J. Austin Cottrell, Thomas J. R Hughes, Isogeometric Analysis: Toward Integration of CAD and FEA, 2009. doi:978-0-470-74873-2.

[12] K. A. Johannessen, Optimal quadrature for univariate and tensor product splines, Computer Methods in Applied Mechanics and Engineering 316 (2017) 84 - 99, special Issue on Isogeometric Analysis: Progress and Challenges. doi:http://doi.org/10.1016/j.cma.2016.04.030.

[13] C. Adam, T. Hughes, S. Bouabdallah, M. Zarroug, H. Maitournam, Selective and reduced numerical integrations for NURBS-based isogeometric analysis, Computer Methods in Applied Mechanics and Engineering 284 (2015) 732-761. doi:10.1016/j.cma.2014.11.001.

[14] J. Caseiro, R. Valente, A. Reali, J. Kiendl, F. Auricchio, R. Alves de Sousa, Assumed natural strain NURBS-based solid-shell element for the analysis of large deformation elasto-plastic thinshell structures, Computer Methods in Applied Mechanics and Engineering 284 (2015) 861-880. doi:10.1016/j.cma.2014.10.037.

[15] R. Echter, B. Oesterle, M. Bischoff, A hierarchic family of isogeometric shell finite elements, Computer Methods in Applied Mechanics and Engineering 254 (2013) 170-180. doi:10.1016/j.cma.2012.10.018.

[16] D. Magisano, L. Leonetti, G. Garcea, Advantages of the mixed format in geometrically nonlinear analysis of beams and shells using solid finite elements, International Journal for Numerical Methods in Engineering 109 (9) (2017) 1237-1262. doi:10.1002/nme.5322.

[17] A. Madeo, F. S. Liguori, G. Zucco, S. Fiore, An efficient isostatic mixed shell element for coarse mesh solution, International Journal for Numerical Methods in Engineering n/a (n/a). arXiv:https://onlinelibrary.wiley.com/doi/pdf/10.1002/nme.6526, doi:10.1002/nme.6526. URL https://onlinelibrary.wiley.com/doi/abs/10.1002/nme.6526

[18] L. Leonetti, F. Liguori, D. Magisano, G. Garcea, An efficient isogeometric solid-shell formulation 
for geometrically nonlinear analysis of elastic shells, Computer Methods in Applied Mechanics and Engineering 331 (2018) 159-183. doi:10.1016/j.cma.2017.11.025.

[19] L. Leonetti, F. S. Liguori, D. Magisano, J. Kiendl, A. Reali, G. Garcea, A robust penalty coupling of non-matching isogeometric kirchhoff-love shell patches in large deformations, Computer Methods in Applied Mechanics and Engineering 371 (2020) 113289. doi:https://doi.org/10.1016/j.cma.2020.113289.

URL http://www.sciencedirect.com/science/article/pii/S0045782520304746

[20] R. Pfefferkorn, S. Bieber, B. Oesterle, M. Bischoff, P. Betsch, Improving efficiency and robustness of enhanced assumed strain elements for nonlinear problems, International Journal for Numerical Methods in Engineering $\mathrm{n} / \mathrm{a}$ (n/a) in press. arXiv:https://onlinelibrary.wiley.com/doi/pdf/10.1002/nme.6605, doi:https://doi.org/10.1002/nme.6605.

URL https://onlinelibrary.wiley.com/doi/abs/10.1002/nme.6605

[21] L. Leonetti, D. Magisano, F. S. Liguori, G. Garcea, An isogeometric formulation of the Koiter's theory for buckling and initial post-buckling analysis of composite shells, Computer Methods in Applied Mechanics and Engineering 337 (2018) 387 - 410. doi:https://doi.org/10.1016/j.cma.2018.03.037. 